\title{
EDITORIAL
}

\section{Pitfalls in the blackbox approach to chemical analysis of plant extracts and environmental samples}

Many multidisciplinary research projects, particularly those related to biological activities of plant extracts and environmental studies, involve chemical analysis at some stage. There is a growing tendency to treat this aspect of the research as a routine 'black box' affair. Often, a sample is given to the analyst who subjects it to some form of instrumental analytical method (with varying degrees of automation) and the output is given back to the researcher, who then treats the data as accurate and derives various conclusions; sometimes quite erroneous.

Errors can arise due to lack of awareness of the researcher of the science behind the analytical method. For example, in GCMS analysis for the identification of unknown compounds, the reliability of the results depends not only on instrumental factors but also on data processing methods. It is surprising how often compounds with very low match factors are reported as being present in the sample under study, without any qualification. A more serious source of error, is in not relating the analytical result to the nature of the sample being analysed. As an example, it is not uncommon to find common plasticizers (which probably would have originated from impure solvents used in the extraction process) being reported as plant metabolites. (It is a reflection of the current trend towards compartmentalization of knowledge that consideration of possible bio-synthetic pathways, to novel compounds considered to be isolated from plant materials is no longer fashionable.)

In quantitative analysis, the need for method validation when non-standard methods are used is also often neglected, which brings into question the validity of the conclusions based on the analytical results. With environmental samples in particular, the pre-analytical steps need to carefully chosen so as to avoid artefact formation and matrix effects on the analyte signals. Undoubtably, some of these problems could be avoided if a competent analytical chemist is involved in the project, not only at the point of analysis but also at its planning stage.

It is worthwhile to note that in multidisciplinary research requiring chemical analysis, a black box approach coupled with artificial intelligence which can rapidly generate a large amount of data, while being convenient, cannot substitute for the human intelligence, knowledge and judgement, required to provide a meaningful interpretation of that data. 